\title{
LncRNA RP5-998N21.4 promotes immune defense through upregulation of IFIT2 and IFIT3 in schizophrenia
}

Bo Guo ${ }^{1,2,8}$, Tingyun Jiang ${ }^{3,8}$, Fengchun $\mathrm{Wu}^{4,8}$, Hongyu $\mathrm{Ni}^{1}$, Junping $\mathrm{Ye}^{1}$, Xiaohui $\mathrm{Wu}^{1}$, Chaoying $\mathrm{Ni}^{1}$, Meijun Jiang ${ }^{5}$, Linyan $\mathrm{Ye}^{1}$, Zhongwei $\mathrm{Li}^{1}$, Xianzhen Zheng ${ }^{5}$, Shufen $\mathrm{Li}^{1}$, Qiong Yang ${ }^{4}$, Zhongju Wang ${ }^{1}$, Xingbing Huang ${ }^{4 凶}$ and Cunyou Zhao ${ }^{1,2,6,7 凶}$

Schizophrenia is a complex polygenic disease that is affected by genetic, developmental, and environmental factors. Accumulating evidence indicates that environmental factors such as maternal infection and excessive prenatal neuroinflammation may contribute to the onset of schizophrenia by affecting epigenetic modification. We recently identified a schizophrenia-associated upregulated long noncoding RNA (IncRNA) RP5-998N21.4 by transcriptomic analysis of monozygotic twins discordant for schizophrenia. Importantly, we found that genes coexpressed with RP5-998N21.4 were enriched in immune defense-related biological processes in twin subjects and in RP5-998N21.4-overexpressing (OE) SK-N-SH cell lines. We then identified two genes encoding an interferoninduced protein with tetratricopeptide repeat (IFIT) 2 and 3, which play an important role in immune defense, as potential targets of

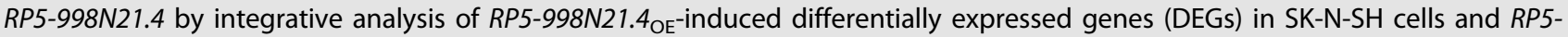
998N21.4-coexpressed schizophrenia-associated DEGs from twin subjects. We further demonstrated that RP5-998N21.4 positively regulates the transcription of IFIT2 and IFIT3 by binding to their promoter regions and affecting their histone modifications. In addition, as a general nuclear coactivator, RMB14 (encoding RNA binding motif protein 14) was identified to facilitate the regulatory role of RP5-998N21.4 in IFIT2 and IFIT3 transcription. Finally, we observed that RP5-998N21.4 ${ }_{O E}$ can enhance IFIT2- and IFIT3mediated immune defense responses through activation of signal transducer and activator of transcription 1 (STAT1) signaling pathway in U251 astrocytoma cells under treatment with the viral mimetic polyinosinic: polycytidylic acid (poly I:C). Taken together, our findings suggest that IncRNA RP5-998N21.4 is a critical regulator of immune defense, providing etiological and therapeutic implications for schizophrenia.

Schizophrenia (2022)8:11; https://doi.org/10.1038/s41537-021-00195-8

\section{INTRODUCTION}

Schizophrenia is a complex genetic disease that affects approximately $1 \%$ of the global population ${ }^{1}$. The complexity of schizophrenia is recognized to result from interactions between the genome and the environment ${ }^{2}$. Epidemiological studies have suggested that prenatal exposure to bacterial or viral infection is an important environmental risk factor for schizophrenia ${ }^{3}$. Accumulating evidence suggests that epigenetic modifications may be particularly vulnerable to environmental influences, especially during embryonic development, and play key roles in mediating the interplay between genomic and environmental factors underlying the development of schizophrenia ${ }^{4}$. As one of the major epigenetic modifications, long noncoding RNAs (IncRNAs), which are $>200 \mathrm{nt}$ in length, play important roles in the regulation of the immune response through different mechanisms, including acting as signals, decoys, guides, or scaffolds ${ }^{5}$. In recent years, several IncRNAs involved in the inflammatory response or viral infection by binding a target protein have been described ${ }^{6,7}$. For instance, the IncRNA IVRPIE promotes the host antiviral immune response by regulating interferon (IFN) $\beta 1$ and IFN-stimulated gene (ISG) expression ${ }^{8}$. The secretion of IFNs from host cells is a response to various pathogens, such as viruses, bacteria, fungi, or parasites, and induces a protective immune defense. IFNs exert their antiviral effects via Janus kinase (JAK)/STAT-mediated signaling through IFN receptors, leading to the induction of approximately 300 ISGs. Several ISG proteins are structurally characterized by tetratricopeptide repeats, and they are called IFN-induced proteins with tetratricopeptide repeats (IFITs). IFIT2 and IFIT3 are IFITs; IFIT3 (ISG60) is also involved in poly I:C-induced CXCL10 expression through the Toll-like receptor 3 (TLR3)/IFN- $\beta / S T A T 1$ axis in U373MG human astrocytoma cells ${ }^{9}$, suggesting that IFITs may play important roles in a variety of biological processes, including physiological innate immunity and pathological inflammation in the central nervous system.

We previously performed transcriptomic analysis of monozygotic twins discordant for schizophrenia and identified two schizophrenia-associated upregulated IncRNAs, AC006129.1 and RP5-998N21.4, whose coexpressed genes are involved in immune and defense response-related biological processes ${ }^{10}$. We delineated the mechanism by which AC006129.1 binds to the promoter region of the transcriptional repressor Capicua (CIC) and promotes DNA methylation-mediated CIC downregulation by facilitating the interactions of DNA methyltransferases with the CIC

\footnotetext{
${ }^{1}$ Department of Medical Genetics, School of Basic Medical Sciences, and Guangdong Technology and Engineering Research Center for Molecular Diagnostics of Human Genetic Diseases, Southern Medical University, Guangzhou, Guangdong, China. ${ }^{2}$ Key Laboratory of Mental Health of the Ministry of Education, Guangdong-Hong Kong-Macao Greater Bay Area Center for Brain Science and Brain-Inspired Intelligence, and Guangdong Province Key Laboratory of Psychiatric Disorders, Southern Medical University, Guangzhou, Guangdong, China. ${ }^{3}$ The Third People's Hospital of Zhongshan, Zhongshan, Guangdong, China. ${ }^{4}$ Department of Psychiatry, the Affiliated Brain Hospital of Guangzhou Medical University (Guangzhou Huiai Hospital), Guangzhou, Guangdong, China. ${ }^{5}$ Guangdong Mental Health Center, Guangdong Provincial People's Hospital, Guangdong Academy of Medical Sciences, Guangzhou, China. ${ }^{6}$ Experimental Education/Administration Center, School of Basic Medical Science, Southern Medical University, Guangzhou, China. ${ }^{7}$ Department of Rehabilitation, Zhujiang Hospital of Southern Medical University, Guangzhou, China. ${ }^{8}$ These authors contributed equally: Bo Guo, Tingyun Jiang, Fengchun Wu.

凶email: hxbing2002@163.com; zhaocunyou@gmail.com
} 
promoter, thereby alleviating $\mathrm{CIC}$-induced suppressor of cytokine signaling 3 (SOCS3) repression. Derepression of SOCS3 enhances the anti-inflammatory response by inhibiting JAK/STAT-signaling activation. However, the underlying gene regulatory mechanism that mediates the roles of another disease-associated IncRNA RP5998 N21.4 in the context of schizophrenia remains unclear.

In this study, we performed integrative RNA sequencing data analysis of monozygotic twin discordant for schizophrenia, a InCRNA RP5-998N21.4 overexpressing SK-N-SH cell line, and public postmortem brain datasets to identify the potential coexpressed target genes of RP5-998N21.4, and we then identified the mechanism by which RP5-998N21.4 underlies the development of schizophrenia by regulating IFIT2- and IFIT3-mediated antiviral immune response pathways.

\section{RESULTS}

Upregulation of RP5-998N21.4 in schizophrenia is involved in immune response-related pathways

We previously found that the IncRNA RP5-998N21.4 (also called ENSG00000234571, NONHSAT225469.1, or NONHSAG105013.1) was upregulated in the patient within monozygotic twins discordant for schizophrenia $\left(\log _{2} \mathrm{FC}=1.58 \text { and } \mathrm{FDR}=0.006\right)^{10}$. We then validated its expression level in blood samples of an independent validation cohort containing 51 schizophrenia patients and 48 nonpsychiatric controls. qPCR analysis revealed significantly increased RP5-998N21.4 expression levels in patients with schizophrenia compared with nonpsychiatric controls (increased by $226 \%, P<0.001$ and $P_{\text {covariate }}=0.029$ when age and sex were included as covariates in ANCOVA; Fig. 1a). Moreover, upregulation of RP5-998N21.4 in blood samples of patients with schizophrenia remained significant in the CommonMind Consortium (CMC) brain RNA-seq dataset $\left(\log _{2} \mathrm{FC}=0.03, P=0.01\right)^{11}$ and met the trend line in the PsychENCODE $\left(\log _{2} \mathrm{FC}=0.11, P=0.17\right)^{12}$ and Children's Hospital of Philadelphia (CHP, Log2FC $=0.20, P=$ $0.48)^{13}$ brain RNA-seq datasets. To explore the biological implications of IncRNA-RP5-998N21.4 in schizophrenia, we identified 367 mRNAs correlated with IncRNA-RP5-998N21.4 expression (|coefficient $r \mid>0.5, P<0.05)$ among the 16 individuals from the same four pairs of schizophrenia-discordant twins (SDC) and four pairs of healthy concordant control twins (HCC) included in the above IncRNA analysis (Supplementary Table 1). We then used a weighted gene coexpression network analysis (WGCNA) approach to perform coexpression analyses of the IncRNA RP5-998N21.4 and these 367 mRNAs in these sixteen individuals and identified that 57 mRNAs were coexpressed with IncRNA RP5-998N21.4 in the brown module (Supplementary Fig. 1 and Supplementary Table 2) and were also significantly enriched in whole blood, minor salivary gland, and spleen tissues based on the GTEx RNA-seq dataset (Supplementary Table 3$)^{14}$. Gene ontology-biological pathway (GO-BP) analysis revealed significant enrichment of the 57 coexpressed genes in terms such as "immune response", "immune effector process", "defense response to virus", "type I IFN signaling pathway", and "response to IFN-beta" (Fig. 1b and Supplementary Data 1), suggesting functional implications of RP5-998N21.4 in the pathogenesis of schizophrenia.

As annotated in the University of California, Santa Cruz (UCSC) database, the RP5-998N21.4 transcript is located on the negative strand of chromosome 1 (chr1:149320440-149379646, hg19) and exists in one isoform of $3540 \mathrm{nt}$ with two exons (Supplementary Fig. 2). The in silico results obtained with the open reading frame (ORF) prediction tool ORFfinder and the Phylogenetic Codon Substitution Frequency tool to distinguish coding and noncoding transcripts consistently showed that RP5-998N21.4 has no potential protein-coding ability (Supplementary Fig. 3). We also observed that RP5-998N21.4 was highly expressed in human white blood cells and the SK-N-SH cell line, as determined by qPCR, and was moderately expressed in whole blood and brain tissues from published NONCODE and GTEx RNA-seq datasets (Supplementary Fig. $4 a-c)^{14,15}$. We then characterized the subcellular localization of RP5-998N21.4 and observed that it was expressed predominantly in the nucleus (Supplementary Fig. 4d), as determined by qPCR in HEK293T cells and RNA-seq data in SK-N-SH cells from the IncATLAS dataset ${ }^{16}$.

To further elucidate the molecular mechanism underlying the upregulation of RP5-998N21.4, we overexpressed a recombinant lentiviral vector containing RP5-998N21.4 in SK-N-SH cells. RNAseq analysis identified 1722 differentially expressed genes (DEGs, DE-seq: $\mid$ LogFC| $>0.5$, FDR < 0.01) (Supplementary Data 2) induced by RP5-998N21.4 $\mathrm{OE}$. GO-BP analysis revealed significant enrichment of the 1722 DEGs in terms such as "neurogenesis", "type I IFN signaling pathway", "defense response to virus", and "response to IFN-beta" (Fig. 1C and Supplementary Data 3), further supporting the role of RP5-998N21.4 related to immune response pathways. We then observed significant overlap (three overlapping genes (IFIT2, IFIT3, and ANXA3; OR $=77.4, P=7.4 \mathrm{e}-5$; Fig. 1d) between RP5-998N21.4 ${ }_{O E}$-induced DEGs in SK-N-SH cells and $R P 5-998 N 21.4-c o e x p r e s s e d$ schizophrenia-associated DEGs in twin subjects. These three genes were upregulated consistently in RP5998N21.4 OE SK cells and in MZ twin patients, and their expression was positively correlated with RP5-998N21.4 expression. Moreover, coexpression patterns of RP5-998N21.4 with IFIT2, IFIT3, and ANXA3 were further observed in whole blood and brain tissue from the GTEx RNA-seq dataset (Supplementary Data 4) ${ }^{14}$. IFIT2 and IFIT3 have been reported to positively regulate TLR3/IFN- $\beta$ /phosphorylation of the STAT1 axis in U373MG human astrocytoma cells ${ }^{9}$ and play important roles in the antiviral response ${ }^{17,18}$. The upregulation of IFIT2 and IFIT3 observed in our twin patients was also observed to be significant in a lymphoblastoid cell lines (LCL) study (IFIT2: $\beta=0.4, \mathrm{FDR}=1.7 \mathrm{e}-12$; IFIT3: $\beta=0.5, \mathrm{FDR}=$ $7.8 \mathrm{e}-15)^{19}$ and to meet the trend line in the CMC brain study (IFIT2: Log2FC $=0.09$ and $P=0.05$; IFIT3: $\log 2 \mathrm{FC}=0.02, P=0.7)^{11}$. $A N X A 3$, as a marker of brain microglia ${ }^{20}$, is reported to be associated with cell death in lactacystin-mediated neuronal injury $^{21}$ and to inhibit the PI3K/Akt signaling pathway ${ }^{22,23}$. The upregulation of $A N X A 3$ observed in our twin patients was also supported by the $\mathrm{LCL}$ study $(\beta=0.2, \mathrm{FDR}=1.4 \mathrm{e}-3)^{19}$ and $\mathrm{a}$ PsychENCODE brain dataset $(\mathrm{LogFC}=0.08 \text { and } \mathrm{FDR}=0.07)^{12}$. Collectively, these results indicate that RP5-998N21.4 might regulate immune defense-related pathways by promoting the transcription of IFIT2, IFIT3, or ANXA3, which are involved in the pathophysiology of schizophrenia.

\section{RP5-998N21.4 promotes IFIT3 and IFIT2 transcription by affecting chromatin modifications}

We further explored the mechanism involved in RP5-998N21.4mediated regulation of IFIT2, IFIT3, and ANXA3 expression. It has been reported that IncRNAs can act as scaffolds by interacting with protein partners to facilitate their binding to target genes to induce their transcription ${ }^{7}$. We first confirmed that RP5-998N21.4 overexpression (RP5-998N21.4 OE) increased IFIT2 and IFIT3 expression levels in HEK293T and SK-N-SH cells and increased the ANXA3 expression level only in SK-N-SH cells (Fig. 2a, b). We then used LongTarget to predict several binding sites of RP5$998 N 21.4$ in the proximal promoter regions of IFIT2 and IFIT3 and validated the upregulation of IFIT2 and IFIT3 expression by RP5998N21.4 $\mathrm{OE}$ in HEK293T cells cotransfected with pCDNA-RP5998 N21.4 and a luciferase reporter vector driven by the IFIT2 or IFIT3 promoter (Fig. 2c).

Next, we tested the binding activities of RP5-998N21.4 to the IFIT2 or IFIT3 promoter region using chromatin isolation by RNA purification (ChIRP) assay and observed that RP5-998N21.4 can bind to the proximal promoter regions of IFIT2 and IFIT3, as determined by qPCR analysis of the retrieved DNA (Fig. 2d) or RNA 
a

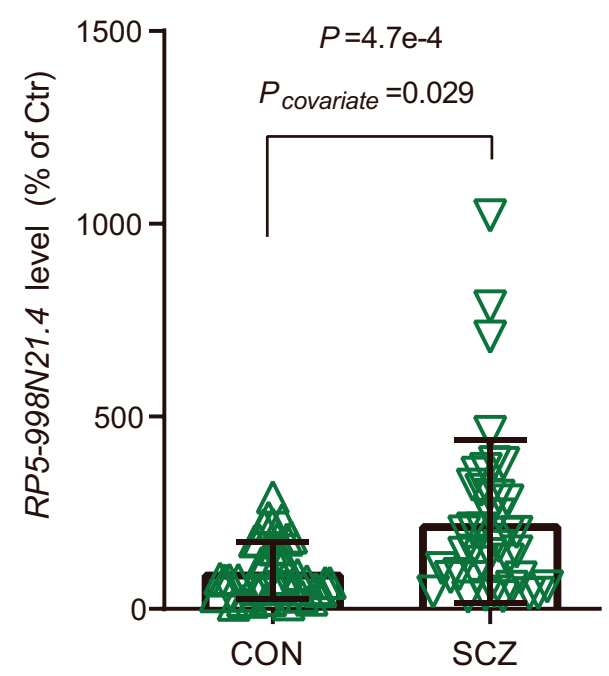

b

Defense response to other or Immune response Immune organism Response to other organism

Response to external biotic stimulus
Response to bion Secretion. Secretion by cell. Defense response

Response to bacterium

Response to virus

Defense response to virus. Innate immune response

Type I interferon signaling pathway Cellular response to type I interferon Response to interferon-beta Response to type I interferon

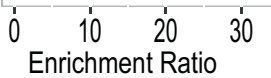

d

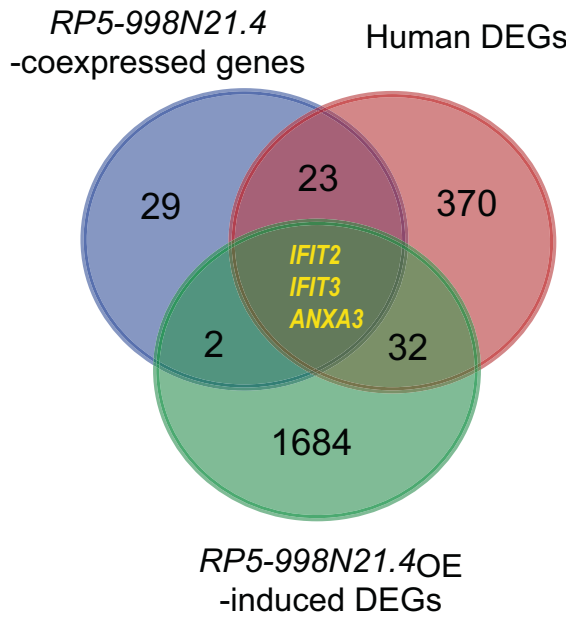

Fig. 1 Upregulation of RP5-998N21.4 in schizophrenia is involved in immune defense-related pathways. a The RP5-998N21.4 level in peripheral blood samples from the validation cohort, namely, 51 patients with schizophrenia (SCZ) and 48 nonpsychiatric controls (CON). The expression levels in SCZ and CON are shown as percentages relative to the mean level in CON with standard deviations (s.d.) indicated by error bars. Significant differences between the two cohorts were determined with ANCOVA without covariates $(P<0.05)$ or with age and sex as covariates (Pcovariate). b, c Functional enrichments of GO-BP annotations among RP5-998N21.4-coexpressed genes in human twin subjects (b) or among RP5-998N21.4 ${ }_{O E}$-induced mouse DEGs (c) are shown for the top gene sets. $\mathbf{d}$ Venn diagram showing overlapping relationships. The numbers indicate the gene counts among the RP5-998N21.4-coexpressed genes in humans, the schizophrenia-associated DEGs (human DEGs) from twin subjects, and the RP5-998N21.4 $\mathrm{OE}$ DEGs from SK-N-SH cells. All blots were derived from the same experiments and were processed in parallel.

(Fig. 2e) from RP5-998N21.4-ChIRP. Finally, we examined whether the binding of RP5-998N21.4 to the IFIT2 or IFIT3 promoter region affects the chromatin state by performing chromatin immunoprecipitation (ChIP)-qPCR for a transcriptionally active histone mark (H3K4me3) and a repressive histone mark (H3K27me3). Notably, H3K4me3 enrichment at the IFIT2 promoter region was significantly higher in RP5-998N21.4 OE HEK293T cells than in control cells (Fig. 2f). In contrast, H3K27me3 enrichment at the IFIT2 promoter region was significantly lower in RP5-998N21.4 $\mathrm{OE}$ cells than in control cells (Fig. $2 \mathrm{~g}$ ). Similarly, marginal alterations in enrichment were also observed for H3K4me3 ( $p=0.051$; Fig. 2f) and H3K27me3 ( $p=0.058$; Fig. $2 \mathrm{~g}$ ) at the IFIT3 promoter region. However, we did not detect significant H3K4me3 and H3K27me3 enrichment at the $A N X A 3$ transcriptional start site region in
RP5-998N21.4 $\mathrm{OE}$ cells and the corresponding control cells (Supplementary Fig. 5), indicating that there may be other mechanisms involved in ANXA3 transcription. In summary, these results indicate that RP5-998N21.4 regulates the transcription of IFIT2 and IFIT3 by binding to their promoter regions and affecting histone modifications in these promoter regions.

\section{RBM14 facilitates the regulatory role of $R P 5-998 N 21.4$ in IFIT2 and IFIT3 transcription}

We next explored the associated proteins that interact with RP5-998N21 using in silico prediction analysis and an in vivo experiment. We first employed LncADeep software to identify 142 proteins that were predicted to significantly interact with RP5-998N21.4 (score $>0.5$, Supplementary Data 5) ${ }^{24}$. We then 

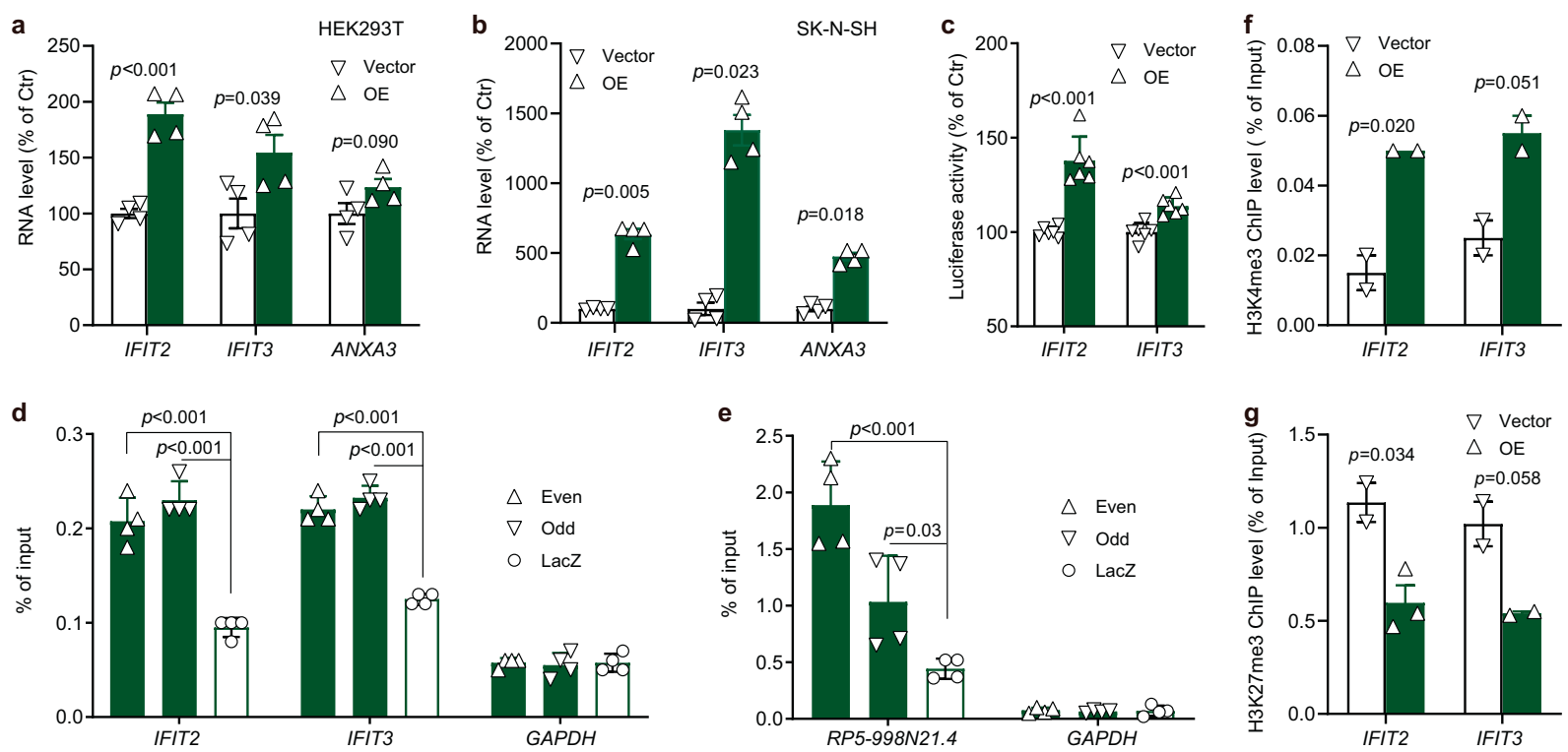

Fig. 2 RP5-998N21.4 regulates its targets through histone modifications. a-c Effects of RP5-998N21.4 $\mathrm{OE}$ on the endogenous expression of potential target genes in HEK293T cells (a) and in SK-N-SH cells in panel (b) or on IFIT2 and IFIT3 promoter activities in luciferase reporter assays in HEK293T cells (c). d, e ChIRP assay of the interaction of IFIT2 or IFIT3 with RP5-998N21.4 in RP5-998N21.4 OE HEK293T cells. The retrieved IFIT2 and IFIT3 DNA (d) or IncRNA-RP5-998N21.4 (e) from the IncRNA RP5-998N21.4 ChIRP assay was quantified by qPCR, and the data in columns are expressed as the percentage of input. Data for the even $(\Delta: \# 2,4,6)$ - and odd $(\nabla: \# 1,3,5)$-numbered probes of $R P 5-998 N 21.4$, as well as the LaCZ probe $(\mathrm{O})$ as the negative control, are shown in the panels. f, $\mathbf{g}$ ChIP-qPCR of H3K4me3 (f) and H3K27me3 (g) enrichment at the promoter regions of IFIT2 and IFIT3 in HEK293T cells with or without RP5-998N21.4 OE. The white column represents the blank control group, and the green column represents the RP5-998N21.4 OE group. The $p$ value from Student's $t$ test is shown between the indicated comparisons. The data are shown with $\Delta$ or $\nabla$ and are presented as the mean \pm s.d. values. All blots were derived from the same experiments and were processed in parallel.

performed an RNA pulldown assay using in vitro-transcribed biotinylated RP5-998N21.4 or its antisense control RNA to pull down protein partners from nuclear extracts of HEK293T cells. RNA-protein complexes were captured with streptavidin magnetic beads, separated by sodium dodecyl sulfate-polyacrylamide gel electrophoresis (SDS-PAGE), and stained with coomassie brilliant blue, and the bands of interest were excised and sent for mass spectrometry analysis (Fig. 3a and Supplementary Fig. $6 a)$. This approach identified 646 proteins that bound significantly to RP5-998N21.4 (peptide expectation value $<0.05$, Supplementary Data 6), and three of the 646 proteins RBM14, CTBP1, and MCM7 were also predicted to bind to RP5-998N21.4 by the above mentioned LncADeep software (Fig. 3b).

We further found that overexpression of RBM14 (Fig. 3c) significantly increased the mRNA level of endogenous IFIT2 (Fig. 3d) and marginally increased that of IFIT3 (Fig. 3e) in HEK293T cells with or without cotransfection of RP5-998N21.4, whereas overexpression of MCM7 and CTBP1 did not increase the endogenous IFIT2 and IFIT3 mRNA levels in RP5-998N21.4 $\mathrm{OE}$ HEK293T cells (Supplementary Fig. 6b, c). We also employed shRNA to achieve knockdown (KD) of endogenous RBM14 expression (Fig. 3f) and observed that RBM14-KD significantly attenuated the increases in IFIT2 (Fig. 3g) and IFIT3 (Fig. 3h) transcription induced by RP5-998N21.4 OE in HEK293T cells. Moreover, RNA immunoprecipitation (RIP) assay using an anti-RBM14 antibody followed by GPCR showed that RP5-998N21.4 was apparently present in the immunoprecipitates of RBM14 from HEK293 cells (Fig. 3i and Supplementary Fig. 7). RBM14 (RNA binding motif protein 14) encodes a ribonucleoprotein that functions as a general nuclear coactivator and an RNA splicing modulator $^{25}$. RBM14 has been identified to be significantly upregulated in postmortem brain tissues in the PsychENCODE database $(\log 2 \mathrm{FC}=0.072, \mathrm{FDR}=0.001)^{12}$ and marginally upregulated in the $L C L$ dataset $(\beta=0.122, F D R=0.098)^{19}$ of patients with schizophrenia compared to nonpsychiatric controls in public RNAseq datasets. These observations suggest that RBM14 facilitates the regulatory role of RP5-998N21.4 in IFIT2 and IFIT3 transcription.

\section{RP5-998N21.4 promotes the immune response by activating STAT1 signaling}

Since IFIT2 and IFIT3 have been reported to play important roles in the antiviral response through the promotion of TLR3/IFN- $\beta / S T A T 1$ axis activity ${ }^{9}$, we then examined whether RP5-998N21.4-mediated upregulation of IFIT2 and IFIT3 activates STAT1 signaling. We first observed that activation of STAT1 was enhanced by RP5-998N21.4induced dose-dependent upregulation of IFIT2 and IFIT3 in HEK293T cells (Fig. 4a and Supplementary Fig. 8). We next used the viral mimetic poly $1: C$ to experimentally model viral infection in U251 astrocytoma cells and evaluated the role of RP5-998N21.4 in the response to infection mediated through upregulation of IFIT3 expression (Supplementary Fig. 9). We observed that poly I:C significantly induced the protein levels of IFIT2, IFIT3, and phosphorylated STAT1 (Fig. 4b and Supplementary Fig. 10), as well as the RNA levels of IFIT2, IFIT3, and CXCL10 (Fig. 4C-e), which were enhanced by RP5-998N21.4 overexpression in U251 cells. These results indicate that RP5-998N21.4 elicits an antiviral response through upregulation of IFIT2- and IFIT3-mediated activation of the STAT1 signaling pathway.

\section{DISCUSSION}

The present findings reveal that upregulation of RP5-998N21.4 promotes the activity of immune response-related pathways through upregulation of IFIT2 and IFIT3 in the context of schizophrenia and that overexpression of this IncRNA can enhance IFIT2- and IFIT3-mediated antiviral defense responses through activation of STAT1 signaling pathways in human U251 
a

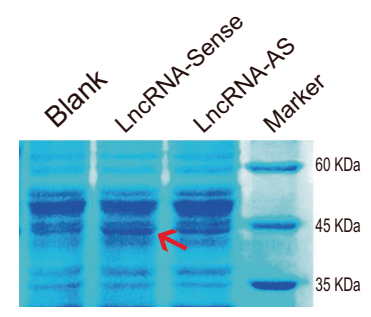

b
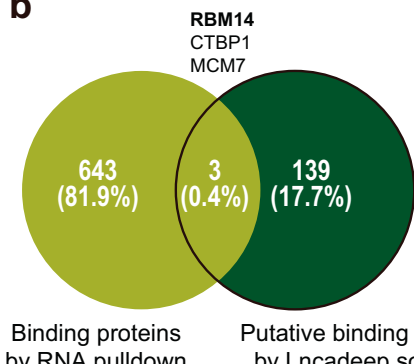

by RNA pulldown

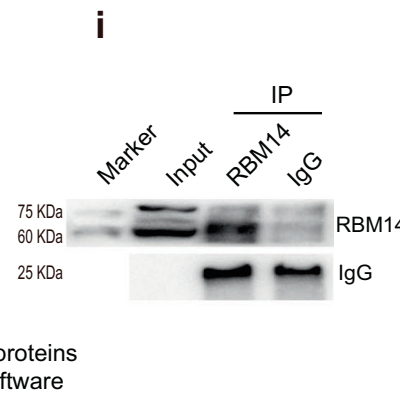

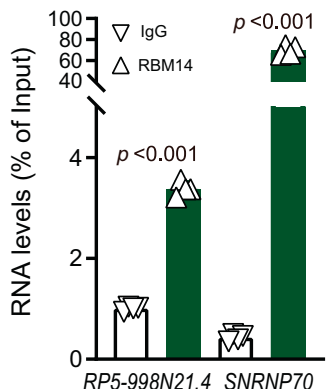

C
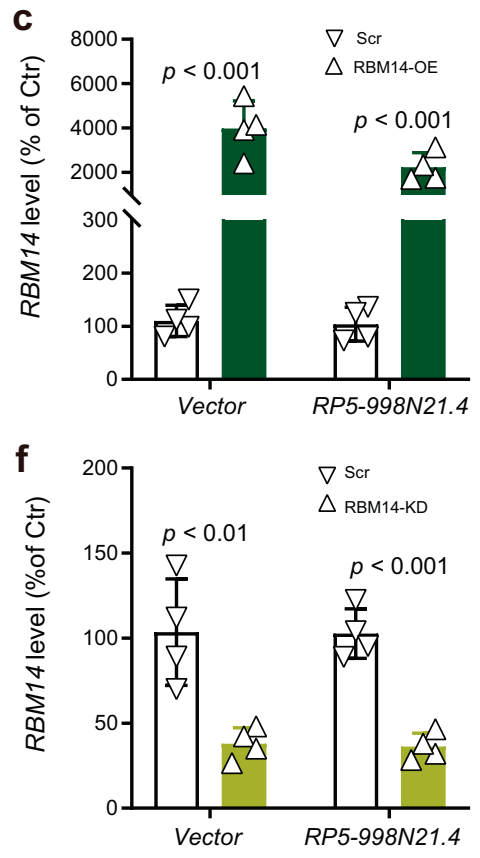

d
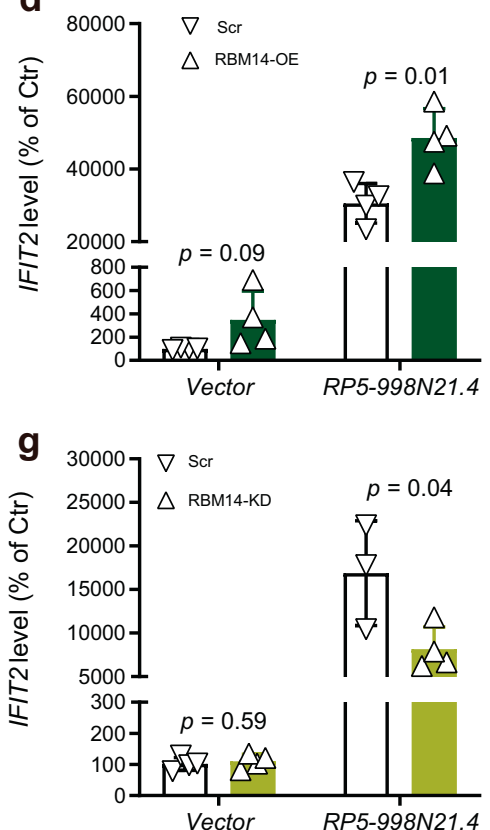

e

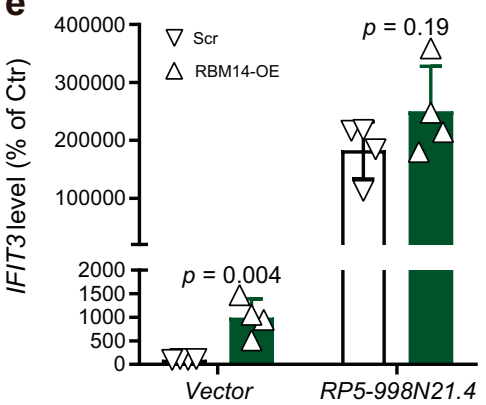

h

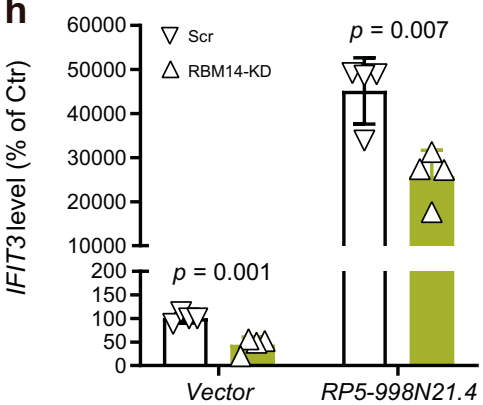

Fig. 3 Effects of the protein interacting partner RBM14 on the regulatory role of RP5-998N21.4 in HEK293T cell. a Coomassie Brilliant Blue staining of the proteins pulled down by PR5-998N21.4 from HEK293T cell lysates; the band indicated by the arrow was sent for mass spectrometry analysis. b Venn diagram of the 646 interacting proteins predicted by LncADeep and the 142 binding proteins identified by mass spectrometry. c-e Effects of RBM14 overexpression (c) on the regulatory role of RP5-998N21.4 in IFIT2 (d) and IFIT3 (e) transcription. f-h Effects of RBM14 knockdown (f) on the regulatory role of RP5-998N21.4 in IFIT2 (g) and IFIT3 (h) transcription. i RNA immunoprecipitation (RIP) combined with qPCR was performed to detect the association of RBM14 with IncRNA-RP5-998N21.4 in HEK293T cells. The immunoblots show the products of immunoprecipitation (IP, left) with the anti-RMB14 antibody, and the qPCR analysis results (right) show that RP5-998N21.4 was abundant in the RBM14 immunoprecipitates of HEK293T cells. Immunoglobin G (IgG) served as the negative immunoprecipitation control, and SNRNP70 served as the positive control for interaction with RBM14. The $p$ value from the two-tailed Student's $t$ test is shown between the indicated two groups. The data from each group are shown with $\Delta$ or $\nabla$ and are presented as the mean \pm s.d. values. All blots were derived from the same experiments and were processed in parallel.

astrocytoma cells treated with the viral mimetic poly I:C. This epigenetic mechanism that links IncRNAs to the promotion of immune defense responses has promising therapeutic implications for schizophrenia.

Schizophrenia is a multifactorial neurodevelopmental disorder with genetic and environmental etiologies. Several studies indicate that prenatal viral/bacterial infections, inflammation, and immune activation increase the offspring's risk for developing schizophrenia. ${ }^{26}$ Recently, IncRNAs have emerged as potential key regulators of inflammatory responses ${ }^{7}$. In our previous study, transcriptomic analysis of monozygotic twins discordant for schizophrenia identified two schizophrenia-associated upregulated IncRNAs, AC006129.1 and RP5-998N21.4; AC006129.1 was identified to reactivate the SOCS3-mediated anti-inflammatory response through DNA methylation-mediated $\mathrm{CIC}$ downregulation in schizophrenia ${ }^{10}$. In this study, we further confirmed that schizophrenia-associated upregulation of RP5-998N21.4 in the patient within SDC twins remained significant in blood samples from an independent sporadic cohort as well as in a previously published CMC postmortem brain RNA-seq dataset ${ }^{11}$. Moreover, we demonstrated that RP5-998N21.4 $\mathrm{OE}_{\text {-induced coexpressed DEGs }}$ in $\mathrm{SK}-\mathrm{N}-\mathrm{SH}$ cell lines were significantly enriched in immune defense-related pathways; two antiviral response-related genes, IFIT2 and IFIT3, were significantly enriched in both RP5-998N21.4coexpressed schizophrenia-associated DEGs and RP5-998N21.4 $\mathrm{OE}^{-}$ induced DEGs and were identified as potential targets of IncRNA RP5-998N21.4. More importantly, consistent with the upregulation of IFIT2 and IFIT3 expression observed in the schizophrenia patients of $M Z$ twin pairs, their expression levels also showed significant alterations in a large sample LCL study ${ }^{27}$, but not in the CMC and PsychENCODE brain studies ${ }^{11}$. Collectively, these results indicate the important role of RP5-998N21.4 in regulating immune response-related pathways involved in the pathogenesis of schizophrenia. 
a
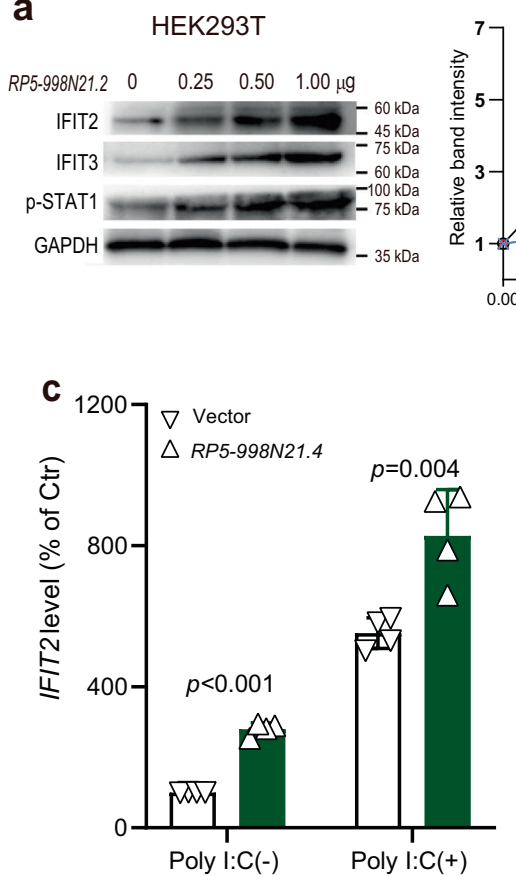

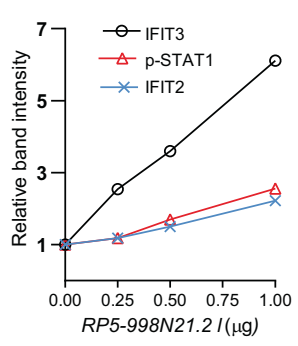

b

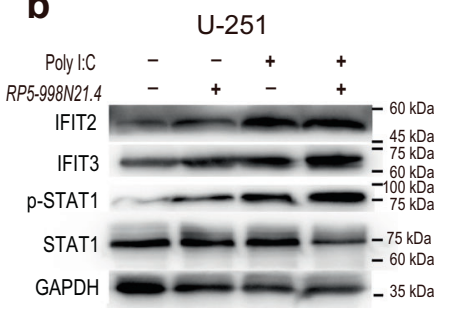

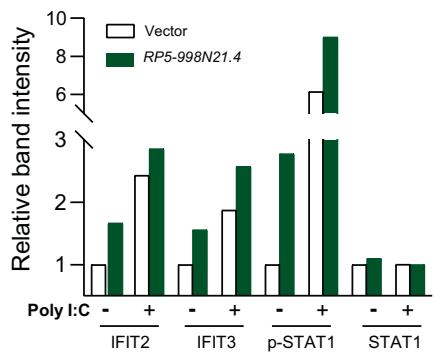

e

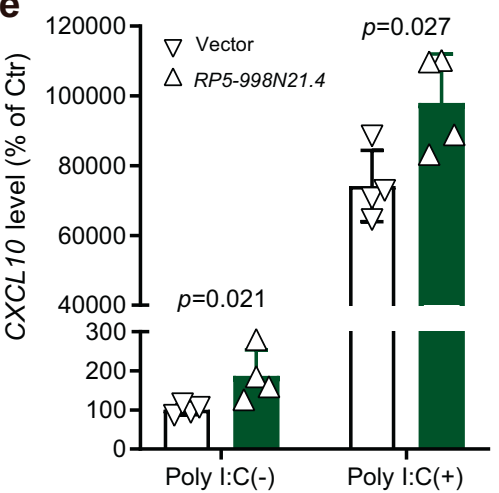

Fig. 4 RP5-998N21.4 promotes immune responses through activation of STAT1 signaling. a Activation of STAT1 signaling was enhanced by RP5-998N21.4-induced dose-dependent upregulation of IFIT2 and IFIT3 in HEK293T cells. Protein extracts from HEK293T cells with RP5998N21.4 $\mathrm{OE}$ were immunoblotted with the indicated antibodies, as shown in the left panel, and the band intensities relative to that of the vector control are shown in the right panel. b-e RP5-998N21.4 enhanced poly I:C-induced upregulation of IFIT2 and IFIT3 and activation of STAT1 signaling in U251 human astrocytoma cells, as determined by western blot (b) and qPCR analyses of IFIT2, IFIT3, and CXCL10 RNA levels (c-e). U251 cells were stimulated with poly l:C $(0,30 \mu \mathrm{g} / \mathrm{ml})$ for another $4 \mathrm{~h}$ and were then collected for immunoblot analysis $44 \mathrm{~h}$ after transfection with or without RP5-998N21.4. Protein extracts from cells transfected with or without RP5-998N21.4 were immunoblotted with the indicated antibodies in the left panel in (b), and the band intensities are shown in the right panel in (b). The $p$ value from the two-tailed Student's $t$ test is shown between the indicated two groups. The data from each group are shown with $\nabla$ (vector control) or $\Delta$ (RP5$\left.998 \mathrm{~N} 21.4_{O E}\right)$ and are presented as the mean \pm s.d. values. All blots were derived from the same experiments and were processed in parallel.

We identified the epigenetic mechanism by which enhancement of IFIT2 and IFIT3 expression by RP5-998N21.4 promotes antiviral defense response pathway activity through the activation of STAT1 signaling pathways in human cells. We demonstrated that RP5-998N21.4 can promote the expression of IFIT2 and IFIT3 by binding to their proximal promoter regions and then affecting their histone modifications. The interactions between RP5998N21.4 and IFIT2 or IFIT3 were revealed by a luciferase reporter assay with a vector containing the RP5-998N21.4-binding regions and by a ChIRP assay. Binding of RP5-998N21.4 at the IFIT2 and IFIT3 promoter regions altered their histone modifications (the activating mark H3K4me3 and repressive mark H3K27me3), further supporting the regulatory role of RP5-998N21.4 in activating IFIT2 and IFIT3 transcription through chromatin remodeling. We further demonstrated that the regulatory role of RP5-998N21.4 in IFIT2 and IFIT3 transcription was facilitated by RBM14. RBM14 has been identified as a component of nuclear paraspeckles $^{28}$ and shown to modulate the transcription and splicing of host genes in response to viral infection ${ }^{29}$. Intriguingly, upregulated IFIT2 and IFIT3 are also involved in the response to viral infection or immune activation and serve as an essential primary barrier to viral infection ${ }^{8,18,30}$. We further demonstrated that STAT1 signaling was activated by RP5-998N21.4-induced upregulation of IFIT2 and IFIT3 in U251 cells under treatment with the viral mimetic poly I:C, in which the $C X C L 10$ expression level was also upregulated. Recently, IFIT2 and IFIT3 were reported to positively regulate the expression of CXCL10 through activation of the TLR3/IFN-beta/STAT1 axis in U373MG cells treated with poly I: $\mathrm{C}^{9}$. CXCL10 is a $\mathrm{C}-\mathrm{X}-\mathrm{C}$ chemokine member that and functions by binding to a specific receptor, CXCR3, and promotes lymphocyte chemotaxis and microglial recruitment. CXCL10 is also reported to be involved in the pathogenesis of Alzheimer's disease and cerebral ischemia, and astrocyte-derived CXCL10 is reported to suppress oligodendrocyte progenitor cell differentiation ${ }^{9}$. Recent studies have also demonstrated altered cytokine activity in schizophrenia, ${ }^{31-34}$ and enhancement of IFIT2 and IFIT3 by RP5998 N21.4 and the subsequent promotion of the immune defense response might partially counteract the defects caused by the disease, with protective or restorative effects against neurotoxicity. Understanding the biological mechanism underlying the regulatory role of $R P 5-998 N 21.4$ in the immune defense response may lead to promising interventions for schizophrenia.

Although our studies demonstrate the involvement of RP5998N21.4 in the development of schizophrenia through the enhancement of IFIT2- and/or IFIT3-mediated immune defense response pathways, it is currently unclear how RP5-998N21.4 influences synapse morphology and function. Furthermore, since most of the function and mechanism study for RP5-998N21.4 in HEK293T cells, neuronal cells, such as primary neurons or neuronlike cells, may be needed to validate the role of RP5-998N21.4 in the regulation of immune defense response in the future.

In conclusion, our findings illustrate an epigenetic mechanism by which upregulation of IncRNA RP5-998N21.4 underlies the development of schizophrenia via the enhancement of IFIT2- and IFIT3-mediated immune defense responses through activation of STAT1 signaling pathways. This epigenetic mechanism that links IncRNAs to the promotion of immune defense responses has promising therapeutic implications for schizophrenia. 


\section{METHODS}

\section{Human subject analysis}

Peripheral blood samples from four pairs of SDC and four pairs of HCC were employed for strand-specific RNA-seq to identify schizophreniaassociated differentially expressed IncRNAs and mRNAs as described ${ }^{10}$. Briefly, DE-IncRNAs and DE-mRNAs were first identified by edgeR pairwise analysis of four schizophrenia cases versus four healthy controls (four SDC twins) and then retained their significant expression differences in case-control analysis of four schizophrenia cases (from four SDC twins) vs. 12 healthy controls (by including four HCC twins). A validation cohort including 51 patients with schizophrenia and 48 unrelated non-psychotic controls was further employed to measure RP5-998N21.4 expression level by using qRT-PCR. All patients meet the diagnostic criteria for schizophrenia according to the fourth edition of the Diagnostic and Statistical Manual of Mental Disorders (American Psychiatric Association). Study participants were free of any diagnosis of mental deficiency, traumatic brain injury, or a history of illicit drug abuse or alcoholism. The control group had no present, past or family history of mental illness or substance abuse. The study was approved by the university review from Southern Medical University and the local medical ethics committees of all participating hospitals and universities and is compliant with the 'Guidance of the Ministry of Science and Technology (MOST) for the Review and Approval of Human Genetic Resources. After introducing the nature of the procedure, the written informed consent of the participants was obtained before the study.

We then used a public RNA-seq dataset to validate the expression differences of interested IncRNA or mRNA between schizophrenia and controls from postmortem brain of CMC dorsolateral prefrontal cortex RNA-seq data ${ }^{11}$ and CHP amygdala RNA-seq data ${ }^{13,35}$ and PsychENCODE RNA-seq dataset (http://www.psychencode.org) ${ }^{12}$, or LCLs of 514 schizophrenia and 690 controls ${ }^{19,27}$

LncRNA RP5-998N21.4 co-expressed network analysis was performed with WGCNA in the $\mathrm{R}$ package ${ }^{36}$ using FPKM values of IncRNA RP5998 N21.4 and mRNAs that displayed significant correlations with RP5998N21.4 (|coefficient $\mathrm{R} \mid>0.5$ and $P<0.05$ ) among these sixteen individuals, and network construction and module detection were analyzed with the "BlockwiseModules" function in the WGCNA package. Coexpression patterns of IncRNA RP5-998N21.4 with the candidate mRNAs were further validated with RNA-seq datasets from brain or blood tissues among at least 40 samples of the GTExv7project (https://dbgap.ncbi.nlm.nih.gov) ${ }^{14}$ by using cor function in R (v3.5.1). GO-BP enrichment analyses were performed using WEB-based GEne SeT AnaLysis Toolkit (WebGestalt: http://www.webgestalt.org/option.php) ${ }^{37}$

\section{RNA-seq analysis of RP5-998N21.4 overexpressed SK-N-SH cells}

To explore the potential targets of IncRNA-RP5-998N21.4, we overexpressed a recombinant lentivirus carrying IncRNA-RP5-998N21.4 in SK-N-SH cells and then performed RNA-seq analysis to identify the DEmRNAs induced by the overexpression of RP5-998N21.4. The recombinant lentivirus driven by the EF1a promoter was constructed by cloning the cDNA sequence of RP5-998N21.4 (Chr1:149372292-149379646, hg19) into the PHAGE-fullEF1a-MCS-IZS Green expression vector via the NhEl and BamHI restriction sites. An empty lentivirus vector with no insert was used as a negative control. To obtain the lentiviruses, the transfer lentiviral plasmid was cotransfected into HEK293T cells with the packaging plasmids pMD2.G and psPAX2 using the $\mathrm{CaPO}_{4}$ coprecipitation method as previously described ${ }^{38}$. Transduction of viral particles into SK-N-SH cells was performed according to the manufacturer's protocol. RNA extracted from the transduced SK-N-SH cells using TRIzol reagent (Invitrogen) with a minimum RNA integrity value of seven was used to build an RNA library. The library was sequenced to a depth of $\sim 27$ million 150-bp paired-end reads per sample on an Illumina HiSeq $X$ ten by Novogene Solution (Tianjin, China). The raw reads were subjected to quality control with FastQC, and clean reads generated from the raw reads with Trimmomatic were mapped to the hg19 reference genome using Bowtie2. Differential expression tests were performed using DEseq2 in the R package.

\section{Cell culturing, plasmid construction, quantitative PCR, Western blotting, and ChIP}

Human embryonic kidney (HEK) 293 T cells, human neuroblastoma cells (SK-N-SH), and human U251 astrocytoma cells were cultured at $37{ }^{\circ} \mathrm{C}$ and $5 \% \mathrm{CO}_{2}$ concentrations in DMEM supplemented with $10 \%$ fetal bovine serum (EX CELL) and antibiotics (penicillin and streptomycin, Gibco, USA). The U251 cells were stimulated with $30 \mu \mathrm{g} / \mathrm{ml}$ poly I:C (dissolved in PBS, ThermoFisher, USA) for $4 \mathrm{~h}$ and collected for further analysis $44 \mathrm{~h}$ after transfection with plasmid using Lipofectamine 2000 (Life Technologies, USA).

The CDNAs from RP5-998N21.4, RBM14, CMC7, and CTBP1 genes were cloned into the pcDNA 3.1(+) plasmid. RBM14-shRNAs were synthesized by Sangon Biotech (China) and cloned into a pLKO.1 vector via the Agel/EcoRl restriction enzyme sites. The IFIT2 (chr10: 91061628-91061686, hg19) and IFIT3 (chr10: 91087690-91087750, hg19) proximal promoter sequences were amplified from human genomic DNA and cloned into a pGL4.18 dualluciferase vector (Promega, USA) via the Kpnl/Xhol (for IFIT2) or Kpnl/HindIII (for IFIT2) restriction sites. These reporter constructs were transiently cotransfected into HEK293T cells together with the pRL-TK plasmid as an internal control for transfection efficiency using Lipofectamine 2000 reagents (Invitrogen, USA). Cells were harvested $48 \mathrm{~h}$ after transfection, and the dual-luciferase activity (Promega) was measured with the Wallac Victor V 1420 Multilabel Counter (PerkinElmer, USA).

Total RNA extracted from cell lines, human peripheral blood using TRIzol reagent (Life Technologies) was reverse-transcribed into CDNA using a PrimeScript RT Reagent Kit with gDNA Eraser (Takara Japan). A comparative qPCR assay with SYBR green dye-containing SuperArray PCR master mix (YEASEN, China) was performed on an ABI Prism 7900 system (Life Technologies) with ACTB and/or GAPDH as reference genes for the quantification of target gene levels as $2^{-\Delta \Delta C t}$ were subjected to statistical analyses. All primers used in this study were synthesized by Sangon Biotech (Supplementary Table 4). Western blotting was performed with rabbit anti-IFIT2 (1:1000, Proteintech, USA), anti-IFIT3 (1: 1000, Proteintech), anti-STAT1 (1: 1000, Proteintech), anti-phosphorate STAT1 (1: 1000, Proteintech), anti-RBM14 (1: 1000, Abclonal, China), rabbit anti-GAPDH (1: 3000 , Proteintech) antibodies.

ChIP assays were performed with cell extracts from HEK293T cells using anti-H3K4me3 (1: 100, Abclonal) or anti-H3K27me3 (1: 100, Abclonal) antibodies as recommended (EZ-ChIP, Merck, Germany). Cell lysates extracted from HEK293T cells were subjected to ChIP assays using antibodies $48 \mathrm{~h}$ after transfection with pcDNA3.1-RP5-998N21.4 or an empty control vector, and the immunoprecipitated DNA was quantified by qPCR using primers IFIT3, IFIT2, and ANXA3 to evaluate the histone modification levels of $\mathrm{H} 3 \mathrm{~K} 4 \mathrm{me} 3$ and $\mathrm{H} 3 \mathrm{~K} 27 \mathrm{me} 3$ (Abclonal) at the target regions.

\section{RNA pulldown assay and mass spectrometry}

To explore the protein partners that interact with RP5-998N21, we performed in silico predictions and an in vivo experiment. We first employed LncADeep software, which is based on deep learning algorithms $\mathrm{s}^{24}$, to predict IncRNA-protein interactions and obtain functional annotations for IncRNAs. We then performed an RNA pulldown assay using in vitro-transcribed biotinylated RP5-998N21.4 or its antisense control RNA to pull down protein partners from HEK293T cell lysates. RNA-protein complexes were captured by streptavidin magnetic beads, separated by SDS-PAGE, and stained with Coomassie Brilliant Blue (Supplementary Fig. 6a). The bands of interest were then excised and sent for mass spectrometry analysis. Briefly, biotin-labeled IncRNAs were transcribed in vitro using a Ribo RNAmax-T7 Biotin Labeling Transcription Kit (RiboBio, China). Biotinylated sense or antisense IncRNA-RP5998 N21.4 was incubated with HEK293T cell lysates at $4^{\circ} \mathrm{C}$ for $2 \mathrm{~h}$. The interacting complexes were then purified with streptavidin agarose beads (S1420, New England Biolabs, USA). The eluted proteins were separated by SDS-PAGE and stained with Coomassie Brilliant Blue, and the interesting protein bands were excised for in-gel trypsin digestion prior to liquid chromatography (LC)-tandem mass spectrometry (MS)/MS analysis at Guangzhou Fitgene Biotechnology Company (China). Proteins with a peptide expectation value $<0.05$ were considered potential IncRNA-RP5-998N21.4 interacting partners.

\section{ChIRP assay}

We employed ChIRP to examine the interaction of IncRNA RP5-998N21.4 with the IFIT2 and IFIT3 promoter region using an EZMagna ChIRP RNA Interactome Kit (Merck). Briefly, HEK293T cell lysates were cross-linked with $1 \%$ glutaraldehyde $48 \mathrm{~h}$ after transfection with pcDNA3.1-RP5-998N21.4 and then sonicated using a Qsonica instrument (USA). A total of ten biotinylated tilling probes were employed to capture InCRNA-RP5-998N21.4 by hybridization with the sonicated chromatin in two separate reactions: 
one for odd-number probes (\#1, 3, 5, 7, and 9) and another for evennumbered probes ( $\# 2,4,6,8$, and 10 ) synthesized by RiboBio technologies (RiboBio, China). The chromatin complexes associated with IncRNA-RP5998N21.4 were pulled down using streptavidin-conjugated magnetic beads and used for RNA and bound DNA isolation. The levels of IncRNA-RP5998 N21.4 obtained from isolated RNA were quantified by qRT-PCR. The levels of bound IFIT2 and IFIT3 DNA from isolated DNAs were quantified by qPCR using primers in the predicted IFIT2 and IFIT3-binding regions.

\section{RIP assay}

RIP experiments were performed in cell extracts isolated from HEK 293 T cells transfected with pcDNA3.1-RP5-998N21.4. Nuclear extracts were immunoprecipitated with $5 \mu \mathrm{g}$ of an anti-RBM14 antibody (Abcam, England) or isotype-matched control IgG overnight. RNA-proteinantibody complexes were captured using Protein A/G Dynabeads (Merck). RNA was eluted in accordance with the manufacturer's instructions. CDNA was synthesized from eluted RNA using a HiScript 1st Strand cDNA Synthesis Kit (Vazyme, China) and analyzed by qPCR.

\section{Reporting summary}

Further information on research design is available in the Nature Research Reporting Summary linked to this article.

\section{DATA AVAILABILITY}

All data generated in the study are included in the article or uploaded as supplementary materials. RNA-Sequencing data of RP5-998N21.4 overexpressed SK-NSH cells have been deposited to the NCBI GEO site with the GEO number GSE186598. The source data of Fig. 3b are provided in Supplementary Data 5 and 6. Additional information is also available upon reasonable request to the corresponding authors.

\section{CODE AVAILABILITY}

Code used for data processing and analysis is available on request from the corresponding author.

Received: 26 July 2021; Accepted: 26 November 2021; Published online: 01 March 2022

\section{REFERENCES}

1. Millan, M. J. et al. Altering the course of schizophrenia: progress and perspectives. Nat. Rev. Drug Discov. 15, 485-515 (2016).

2. Van Os, J., Kenis, G. \& Rutten, B. P. The environment and schizophrenia. Nature 468, 203-212 (2010)

3. Feigenson, K. A., Kusnecov, A. W. \& Silverstein, S. M. Inflammation and the two-hit hypothesis of schizophrenia. Neurosci. Biobehav. Rev. 38, 72-93 (2014).

4. Heyn, H. A symbiotic liaison between the genetic and epigenetic code. Front. Genet. 5, 113 (2014).

5. Chew, C. L., Conos, S. A., Unal, B. \& Tergaonkar, V. Noncoding RNAs: master regulators of inflammatory signaling. Trends Mol. Med. 24, 66-84 (2018).

6. Lin, $\mathrm{H}$. et al. The long noncoding RNA Lnczc3h7a promotes a TRIM25-mediated RIG-I antiviral innate immune response. Nat. Immunol. (2019).

7. Mathy, N. W. \& Chen, X. M. Long non-coding RNAs (IncRNAs) and their transcriptional control of inflammatory responses. J. Biol. Chem. 292, 12375-12382 (2017).

8. Zhao, L. et al. A long non-coding RNA IVRPIE promotes host antiviral immune responses through regulating interferon $\beta 1$ and ISG expression. Front. Microbiol. 11, 2382 (2020).

9. Imaizumi, T. et al. Interferon-stimulated gene (ISG) 60, as well as ISG56 and ISG54, positively regulates TLR3/IFN-beta/STAT1 axis in U373MG human astrocytoma cells. Neurosci. Res. 105, 35-41 (2016).

10. $\mathrm{Ni}$, C. et al. LncRNA-AC006129.1 reactivates a SOCS3-mediated anti-inflammatory response through DNA methylation-mediated $\mathrm{CIC}$ downregulation in schizophrenia. Mol Psychiatry https://doi.org/10.1038/s41380-020-0662-3 (2020).

11. Fromer, M. et al. Gene expression elucidates functional impact of polygenic risk for schizophrenia. Nat. Neurosci. 19, 1442-1453 (2016).

12. Gandal, M. J. et al. Transcriptome-wide isoform-level dysregulation in ASD, schizophrenia, and bipolar disorder. Science 362, 1265 (2018).

13. Tian, T. et al. The long noncoding RNA landscape in amygdala tissues from schizophrenia patients. EBioMedicine 34, 171-181 (2018).
14. Consortium, G. T. et al. Genetic effects on gene expression across human tissues. Nature 550, 204-213 (2017).

15. Fang, S. et al. NONCODEV5: a comprehensive annotation database for long noncoding RNAs. Nucleic Acids Res. 46, D308-D314 (2018).

16. Mas-Ponte, D. et al. LncATLAS database for subcellular localization of long noncoding RNAs. RNA 23, 1080-1087 (2017).

17. Zhou, X. et al. Interferon induced IFIT family genes in host antiviral defense. Int. J. Biol. Sci. 9, 200-208 (2013).

18. Xu, F. et al. NF-kappaB-dependent IFIT3 induction by HBx promotes hepatitis B virus replication. Front. Microbiol. 10, 2382 (2019).

19. Duan, J. et al. Transcriptomic signatures of schizophrenia revealed by dopamine perturbation in an ex vivo model. Transl. Psychiatry 8, 158 (2018).

20. Junker, $\mathrm{H}$. et al. Proteomic identification of an upregulated isoform of annexin $A 3$ in the rat brain following reversible cerebral ischemia. Glia 55, 1630-1637 (2007).

21. Chong, K. W. Y. et al. Annexin $A 3$ is associated with cell death in lactacystinmediated neuronal injury. Neurosci. Lett. 485, 129-133 (2010).

22. Wu, X.-M. et al. MicroRNA-339-3p alleviates inflammation and edema and suppresses pulmonary microvascular endothelial cell apoptosis in mice with severe acute pancreatitis-associated acute lung injury by regulating Anxa3 via the Akt/ mTOR signaling pathway. J. Cell. Biochem. 119, 6704-6714 (2018).

23. $\mathrm{min}, \mathrm{X}$.-I. et al. miR-18b attenuates cerebral ischemia/reperfusion injury through regulation of ANXA3 and PI3K/Akt signaling pathway. Brain Res. Bull. 161, 55-64 (2020).

24. Yang, C. et al. LncADeep: an ab initio IncRNA identification and functional annotation tool based on deep learning. Bioinformatics 34, 3825-3834 (2018).

25. Simon, N. E., Yuan, M. \& Kai, M. RNA-binding protein RBM14 regulates dissociation and association of non-homologous end joining proteins. Cell Cycle 16, 1175-1180 (2017)

26. Muller, N. Inflammation in schizophrenia: pathogenetic aspects and therapeutic considerations. Schizophr. Bull. 44, 973-982 (2018).

27. Sanders, A. R. et al. Transcriptome sequencing study implicates immune-related genes differentially expressed in schizophrenia: new data and a meta-analysis. Transl. Psychiatry 7, e1093 (2017).

28. Jang, Y. et al. Intrinsically disordered protein RBM14 plays a role in generation of RNA:DNA hybrids at double-strand break sites. Proc. Natl Acad. Sci. USA 117, 5329-5338 (2020)

29. Beyleveld, G. et al. Nucleolar relocalization of RBM14 by influenza A virus NS1 protein. mSphere 3, e00549-18 (2018).

30. Ashley, C. L., Abendroth, A., McSharry, B. P. \& Slobedman, B. Interferonindependent upregulation of interferon-stimulated genes during human cytomegalovirus infection is dependent on IRF3 expression. Viruses 11, 246 (2019).

31. Richard, M. D. \& Brahm, N. C. Schizophrenia and the immune system: pathophysiology, prevention, and treatment. Am. J. Health Syst. Pharm. 69, 757-766 (2012).

32. Drexhage, R. C. et al. An activated set point of T-cell and monocyte inflammatory networks in recent-onset schizophrenia patients involves both pro- and antiinflammatory forces. Int J. Neuropsychopharmacol. 14, 746-755 (2011).

33. Jones, K. A. \& Thomsen, C. The role of the innate immune system in psychiatric disorders. Mol. Cell Neurosci. 53, 52-62 (2013).

34. Kirkpatrick, B. \& Miller, B. J. Inflammation and schizophrenia. Schizophr. Bull. 39, 1174-1179 (2013)

35. Liu, Y. et al. Non-coding RNA dysregulation in the amygdala region of schizophrenia patients contributes to the pathogenesis of the disease. Transl. Psychiatry 8, 44 (2018).

36. Langfelder, P. \& Horvath, S. WGCNA: an R package for weighted correlation network analysis. BMC Bioinforma. 9, 559 (2008).

37. Wang, J., Duncan, D., Shi, Z. \& Zhang, B. WEB-based GEne SeT AnaLysis Toolkit (WebGestalt): update 2013. Nucleic Acids Res. 41, W77-W83 (2013).

38. Tiscornia, G., Singer, O. \& Verma, I. M. Production and purification of lentiviral vectors. Nat. Protoc. 1, 241-245 (2006).

\section{ACKNOWLEDGEMENTS}

We thank the individuals for their willingness to participate in this study. Financial supports from the National Natural Science Foundation of China [grant Nos. 81871049, 82002237, and 82101577]; China Postdoctoral Science Foundation [grant No. 2020M682806]; and the Guangdong Science and Technology Foundation [grant Nos. 2019B030316032 and 2017A050506026] are gratefully acknowledged.

\section{AUTHOR CONTRIBUTIONS}

Conceived and designed the experiments: B.G., T.J., F.W., X.H., and C.Z. Performed the experiments: B.G., H.N., C.N., L.Y., and S.L. Analyzed the data: B.G., J.Y., F.W., X.W., Z.L., 
and Z.W. Collected and diagnosed the subjects: T.J., F.W., M.J., X.Z., Q.Y., and X.H. Wrote the paper: B.G. and C.Z. B.G. and T.J. contributed equally to this study. All authors read and approved the final paper

\section{COMPETING INTERESTS}

The authors declare no competing interests.

\section{ADDITIONAL INFORMATION}

Supplementary information The online version contains supplementary material available at https://doi.org/10.1038/s41537-021-00195-8.

Correspondence and requests for materials should be addressed to Xingbing Huang or Cunyou Zhao.

Reprints and permission information is available at http://www.nature.com/ reprints
Publisher's note Springer Nature remains neutral with regard to jurisdictional claims in published maps and institutional affiliations.

(i) Open Access This article is licensed under a Creative Commons Attribution 4.0 International License, which permits use, sharing, adaptation, distribution and reproduction in any medium or format, as long as you give appropriate credit to the original author(s) and the source, provide a link to the Creative Commons license, and indicate if changes were made. The images or other third party material in this article are included in the article's Creative Commons license, unless indicated otherwise in a credit line to the material. If material is not included in the article's Creative Commons license and your intended use is not permitted by statutory regulation or exceeds the permitted use, you will need to obtain permission directly from the copyright holder. To view a copy of this license, visit http://creativecommons. org/licenses/by/4.0/.

(c) The Author(s) 2022 